\title{
The Role of Analytical Chemistry in Agricultural Research: Main goals, Challenges, Demands
}

\author{
Hans Jörg Bachmann*, Thomas Bucheli, Jean Paul, Hans Stünzi, and Hans-Ruedi Bosshard
}

\begin{abstract}
Analytical chemistry has always played an important role in agricultural research, but its objectives and contributions have changed over time. In this paper a description of the historical development is given. Particularities of agricultural analytical chemistry are characterised and the currently used methods for inorganic and organic trace analysis as well as examples of their applications are reported.
\end{abstract}

Keywords: Agricultural research · Analytical chemistry · Organic trace analysis · Reference methods

\section{History}

Since the dawn of true agricultural research in the second half of the nineteenth century, analytical chemistry has played a major supporting role and continues to do so. However, very interesting changes in the objectives and contributions of analytical chemistry have occurred.

One of the main involvements of analytical chemistry has always been to protect farmers from fraud when buying fertilisers and other auxiliary agricultural products. Thus, legal authorities have published ordinances which define not only the active components in a product, but also their concentrations. Compliance was officially controlled in analytical chemistry laboratories. For new products which did not belong to any of the known categories, proof of usefulness was demanded.

This leads us to a further important field of activity of analytical chemistry in agricultural research. Laboratory results were crucial to the understanding of the process-

\footnotetext{
*Correspondence: H.J. Bachmann

Swiss Federal Research Station for Agroecology and Agriculture (FAL),

Reckenholzstrasse 91

Postfach

$\mathrm{CH}-8046$ Zürich

Tel.: +411377 7150

Fax: +411377 7201

E-Mail: hans-joerg.bachmann@fal.admin.ch
}

es and fluxes of substances in land use systems. In the first hundred years the emphasis was on higher yields to meet the increasing demand for food of a growing population. As a result, analytical chemistry remained largely within the framework of agricultural research and production. The laboratory results were used as the basis for determining practical agricultural procedures.

In the second half of the twentieth century, the aim shifted towards optimisation with the relation between costs and yield as the decisive factor of agricultural policy. Furthermore, the necessity of protecting the environment from undesirable effects of agricultural practices was recognised. A good example of this evolution is the coupling of soil analyses to optimal fertilisation on a large scale level, taking into account the demands for environmental protection. To deal with this demand, analytical chemists were asked to develop reliable methods for the efficient measurement of thousands of samples.

A new era for analytical chemistry began in the last third of the twentieth century when the concept of environmental protection became widespread and preservation of soil fertility became a declared goal of agricultural research. The challenges were demanding but fascinating: to detect the pollutants analytical methods were needed with concentration ranges several orders of magnitude below those of traditional agricultural chemistry. In the case of inorganic pollutants, a useful analytical methodology has been developed, but this is not yet true for the determination of organic pollutants.

A further significant change has come with the transfer of chemical analyses to private laboratories, which has led to a sharing of the workload. Thus, the analytical chemists of the research stations were asked to develop and make available to all, reference methods which allowed private laboratories to offer competitive analytical services for most of the analyses which were legally required. As a control of their proficiency and in order to obtain official recognition, the service laboratories have to prove their competence through participation in interlaboratory trials.

\section{Particularities of Agricultural Analytical Chemistry}

Agricultural research sets particular demands on analytical chemistry. For example, the total concentration of an element is often of minor interest in comparison to the fraction of an element which is available to the plants under specific conditions. Thus, the concentration of the analyte is determined in the extract after extraction of the sample with an adequate solvent under given conditions. This can be regarded as the typical model for a consensual method. Its results are defined solely by the method itself and cannot be checked otherwise. Interlaboratory comparisons are usually the only possibility to develop and test this kind of method. In contrast, there are in most cases several procedures and methods to 
determine and validate total concentrations in samples. We may further specify that all analytical results for soils - particularly those from consensual methods - acquire their relevance from a scheme of interpretation which is usually based on agricultural parameters (yield, growth, ...) and plant analyses.

Another particularity is the required long-term comparability of analytical results in field experiments that can last many decades with long periods of crop rotation. The analytical results, obtained periodically during these time spans, must remain comparable, despite possible changes in analytical instrumentation, laboratory reorganisations and retirement of qualified personnel. Monitoring projects, such as the Swiss National Soil Observation network (NABO), make even more heavy demands on long-term comparability due to their duration and increased precision requirements necessary to detect subtle trends.

There is also an obligation for efficient and economical procedures. This tendency is even more important after the transfer of routine analyses to private service laboratories.

\section{Methods of Inorganic Analytical Chemistry in Agricultural Research}

Many of the currently used inorganic analytical methods in agricultural research are based on physical methods of detection. Exceptions are electrochemical methods for the determination of $\mathrm{pH}$ and fluoride in soil extracts and mineralisations which require the use of electrodes. Another exception is the determination of nitrogen according to the methods of Dumas or Kjeldahl. But in the case of plant samples these chemical methods can be replaced by the more efficient technique of near-infrared spectroscopy (NIR).

In the compilation of the reference methods of the Swiss agricultural research stations, the methods most frequently mentioned are photometry and atomic absorption spectrometry with either flame (F-AAS) or electrothermal atomisers (ETA-AAS). These techniques are relatively simple and robust and ample know-how has been accumulated over the years. Furthermore, instrument prices and running costs are moderate enough to allow their acquisition by smaller service laboratories. Even for larger institutes, these methods continue to serve as reference for control checks of individual results which were obtained with more modern and efficient instruments.

Atomic emission spectrometry with inductively coupled plasma (ICP-AES) has become the true work horse for fully automated multi-element analysis. Modern spectrometers with fixed polychromators (simultaneous ICP-AES) are common in larger laboratories due to their robustness, ease of operation as well as bearable buying costs. Axial viewing of the plasma has increased the sensitivity of the instruments allowing simultaneous determination of major and trace elements.

Ultra trace analysis and special work (e.g. semi-quantitative screening analyses or determination of isotope ratios) are almost exclusively performed with mass spectrometry with inductively coupled plasma (ICP-MS). These very sensitive and efficient instruments require a high degree of experience and knowledge and their use is restricted to laboratories dealing with a large number of samples with the above mentioned needs.

\section{Organic Trace Analysis in Agricultural Research}

To perform organic trace analysis at our research station means creating tools which contribute to the realization of our goals, i.e. 1) the development of environmental friendly agricultural systems, and 2) the protection of agriculture from harmful environmental influences. Considering the distribution of organic compounds, agricultural systems play an ambivalent role in as much as they can act both as emitters and receivers of such compounds. Out of the hundreds of thousands of anthropogenic organic compounds currently in use, the most relevant ones agriculturally speaking may be separated in three major groups: a) (ubiquitous) persistent organic pollutants, b) 'classical' pesticides, and c) 'emerging' compounds such as veterinary pharmaceuticals. Among the most prominent representatives of these groups are a) polycyclic aromatic hydrocarbons (PAHs) and polychlorinated biphenyls (PCBs), b) organochlorine pesticides and triazines, and c) veterinary antibiotics such as the tetracyclines. Whereas group b) and c) compounds are usually primarily emitted by agricultural activities, group a) pollutants are introduced into agricultural systems via, e.g. atmospheric deposition or sewage sludges.

Analysis of these pollutants poses a variety of different challenges. For example, within the NABO, we are currently attempting to expand the actual number of observation parameters by the additional analysis of PAHs and PCBs. The analytical method to be developed must be a compromise between a variety of factors such as the large sample number (several hundreds of soil samples for a first overview), low concentrations $(<30 \mu \mathrm{g} / \mathrm{kg}$ for individual PAHs, and $<5 \mu \mathrm{g} / \mathrm{kg}$ for individual PCB congeners), and long-term reproducibility in the order of tens of years. The last factor makes the application of classical Soxhlet extraction preferable to more innovative and sophisticated techniques.

If both PAHs and PCBs, as well as the group b) compounds, are routinely separated and detected by GC/MS, the more modern pesticides, their metabolites, and most of group c) require, because of their higher polarity, the use of liquid chromatography (LC) for separation. In recent years, the development of powerful interfaces have considerably facilitated the coupling of LC with MS, and applications of LC/MS within agricultural research are, as elsewhere, increasing at a rapid rate.

Future challenges for the (agricultural) environmental organic analyst comprise the increasing number of active compounds used widely in agriculture. Even if future agricultural chemicals will be further optimised for higher effectiveness and degradability, a vision which will be probably paralleled by the number of their respective metabolites; their effects may be more subtle and only be visible at long-term scales, especially at sub-acute concentrations levels or in the case of negative synergism of present substances. It is therefore evident that these developments will necessitate highly sensitive detectors and intelligent tools for compound identification.

\section{Conclusions}

From the beginnings till now, analytical chemistry has always played an important role in agricultural research, but its objectives and contributions have changed over time. A general trend to use more specific and sensitive methods, as well as powerful modern and complex instruments, can be observed to satisfy the actual research needs. Since there are no signs to suggest that this trend could change in the future, continuous development will remain an important characteristic of agricultural analytical chemistry.

However, instead of trusting increasingly sophisticated analytical instruments and spending great efforts in the extension of monitoring programs, researchers should think more in terms of processes and attempt to increase their general understanding about fate and behaviour of chemical compounds within the agricultural systems.

Received: May 4, 2002 